\title{
Anti-tumour necrosis factor specific antibody (infliximab) treatment provides insights into the pathophysiology of rheumatoid arthritis
}

\author{
R N Maini, P C Taylor, E Paleolog, P Charles, S Ballara, F M Brennan, M Feldmann
}

Preclinical studies based on in vitro cell systems and in vivo models had established a position for tumour necrosis factor (TNF) $\alpha$ as a pivotal molecule regulating cellular activation and interactions in rheumatoid inflammation by 1992. That neutralisation of $\mathrm{TNF} \alpha$ has a profound impact on the biology of inflammation is reflected by the rapid reduction in the concentration of $\mathrm{C}$ reactive protein (CRP), an acute phase protein, associated with a fall in the level of its well known inducer, interleukin (IL) 6 . This supports the hypothesis that $T N F \alpha$ is a critical part of a regulatory cytokine network.

The reduction in clinical signs of inflammation were soon shown by arthroscopic examination and synovial biopsies of knee joints to be a consequence of reduction in the density of infiltrating lymphocytes and macrophages. Circulating numbers of lymphocytes increased transiently after infliximab in a dose dependent fashion, associated with a reduction in soluble adhesion molecules E selectin, ICAM-1, and density of cells staining for these and VCAM-1 in synovial biopsies. The dynamics of cell trafficking have been examined by tracking the fate of the indium ${ }^{111}$ labelled polymorphonuclear cells injected intravenously in rheumatoid arthritis (RA) patients. These experiments show a reduction in uptake of radioactivity in joints after anti-TNF treatment indicating reduced adhesiveness and retention of the leucocytes in joints.

The results of anti-TNF treatment on the progression of damage to cartilage, bone and other connective tissue components is not yet established in RA, although in collagen induced arthritis in DBA/1 mice, joint protection was reported. Measurement of circulating matrix metalloproteinases, MMP-1 and MMP-3, in their inactive form, has been noted following infliximab in patients. Recently we have documented a reduction in raised concentrations of serum VEGF, an important angiogenic factor after infliximab treatment. More direct evidence of a reduction in angiogenesis has been obtained by synovial biopsies.

In conclusion, the experimental data from anti-TNF blockade in RA indicate that TNF $\alpha$ plays a key part in regulating the proinflammatory cytokine network, cell adhesion, cell migration and angiogenesis in RA joints.

\section{TNF $\alpha$ regulates acute phase protiens and IL6}

The rationale that defined a role for $\mathrm{TNF} \alpha$ as a pivotal molecule regulating cellular activation and interactions in RA via the cytokine cascade had emerged by 1992 from preclinical research. These studies, from our own and other laboratories, were based on rheumatoid tissues in vitro and experimental models of rheumatoid arthritis in vivo. ${ }^{1}$ The critical result, however, that put $\mathrm{TNF} \alpha$ on the map as a valid target for therapeutic manipulation was the first clinical trial with a chimeric (human $\times$ mouse) monoclonal antibody, infliximab (cA2, Remicade), which binds to $\mathrm{TNF} \alpha$ specifically and neutralises its activity. Twenty patients with RA whose disease had proved to be resistant to disease modifying antirheumatoid treatment entered this open label trial in 1992. The results not only demonstrated a lack of toxicity of infliximab, but also strongly hinted at its efficacy as evidenced by a remarkable improvement in clinical signs and symptoms in the majority of treated patients. ${ }^{2}$ The result was made all the more persuasive by the observation that there was an almost immediate reduction in the serum concentrations of CRP and the erythrocyte sedimentation rate (ESR) (fig 1). ${ }^{3}$

As IL6 was already known to be a major regulator of the production of acute phase proteins by hepatocytes, it seemed to us likely that neutralisation of TNF might be mediating its systemic effect by regulating the production of IL6 in RA joints. This hypothesis was confirmed by a post-treatment measurement of circulating IL6 in the initial trial, and subsequently fully substantiated by a study in the placebo controlled setting. ${ }^{4}$ Allowing for diurnal variation observed in the placebo treated patients, marked suppression of circulating CRP and IL6 to near normal levels was demonstrated only in infliximab treated patients. The normalisation of CRP and IL6 was seen at both the low and high dose of anti-TNF but the duration of suppression was dose related (fig 2). Thus, in the group of patients receiving the low dose of infliximab (1 $\mathrm{mg} / \mathrm{kg}$ ) both the CRP and IL6 serum concentrations were suppressed for about two weeks, whereas at the high dose of infliximab (10 $\mathrm{mg} / \mathrm{kg}$ ) suppression of serum IL6 concentrations exceeded four weeks. ${ }^{4}$ Moreover, analysis of the rate of change suggested that the reduction in CRP was preceded by a fall in IL6 levels. The sequence of change (TNF blockade $\rightarrow$ IL6 regulation $\rightarrow$ reduction of CRP production) vividly demonstrated the role of TNF $\alpha$ in the regulation of the pro-inflammatory cascade in RA.

Other serum cytokine levels have been measured and in a subgroup of patients in the placebo controlled trial treated in Erlangen, Germany, a small but significant reduction in
Aspenlea Road,

Hammersmith, London

W6 8LH. 

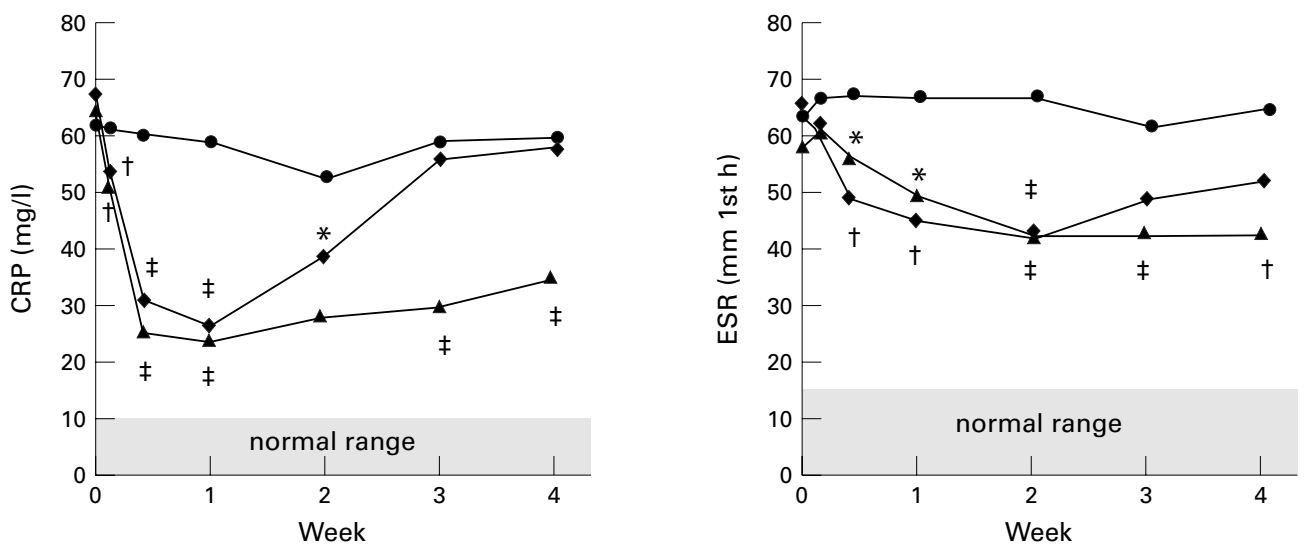

Figure 1 CRP and ESR measurements in a randomised placebo controlled trial of infliximab in RA. Patients were treated on day 0 with a single, two hour infusion of either placebo (circle), $1 \mathrm{mg} / \mathrm{kg}$ infliximab (diamond) or $10 \mathrm{mg} / \mathrm{kg}$ infliximab (triangle). Values are means of 24 patients at each point (25 for $1 \mathrm{mg} / \mathrm{kg}$ group). ${ }^{\star} p<0.05 ;+p<0.01 ; \neq p<0.001$. Reproduced with modification from Lancet 1994;344:1105-10 by kind permission of the editor.
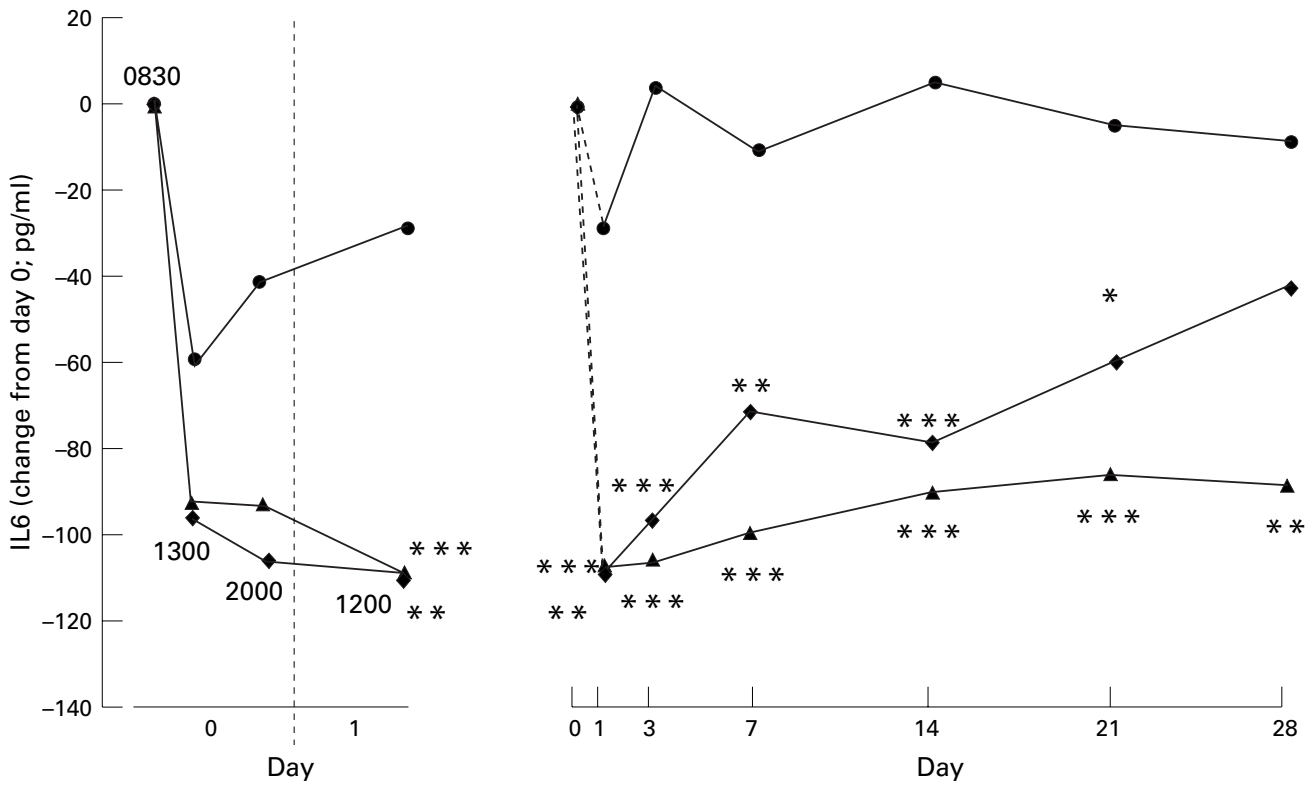

Figure 2 Effect of infliximab on circulating IL6 measurements in a randomised placebo controlled trial of infliximab in $R A$. Patients were treated on day 0 with a single, two hour infusion of either placebo (circle), $1 \mathrm{mg} / \mathrm{kg}$ infliximab (diamond) or $10 \mathrm{mg} / \mathrm{kg}$ infliximab (triangle). A detailed time/response profile on day 0 and 1 is shown on the left, with the mean sampling times indicated on the figure. Changes in circulating IL6 in the same three patient groups over the longer term are shown on the right. Each point represents the median circulating IL6 (pg/ml) concentrations. ${ }^{\star} p<0.05 ;{ }^{\star \star} p<$ $0.01 ;{ }^{\star \star *} p<0.001$ compared with placebo by ANOVA. Reproduced from f Immunol 1999;163:1521-8 by kind permission of the editor.

serum IL1 $\beta$ concentration was observed. ${ }^{5}$ This observation could not be confirmed by serial measurements of barely detectable serum IL1 $\beta$ levels in the full multicentre trial population. ${ }^{4}$ However, a recent analysis of serial synovial biopsies (before and after anti-TNF treatment) has shown a reduction of IL1 $\beta$ production by macrophages in the synovial membrane (Peter Taylor, Ulf Andersson et al unpublished results). These latter data support the proposed action of $\mathrm{TNF} \alpha$ in regulating the production of IL1 in the local milieu of the synovium, but suggest that only a fraction of these proinflammatory cytokines "spill over" into the systemic circulation. The prediction from in vitro experiments that TNF might regulate other proinflammatory cytokines has
Table 1 Anti-TNFa reduces adhesion molecule expression and cellularity in $R A$ tissue

\begin{tabular}{lll}
\hline Parameter & Before infliximab & After infliximab \\
\hline CD3 $^{+}$T cells & $1.6(1.1)$ & $0.6(0.8)$ \\
E selectin & $1.5(0.8)$ & $0.8(0.6)$ \\
ICAM-1 (lining) & $1.0(0.0)$ & $0.4(0.5)$ \\
ICAM-1 (sub-lining) & $1.8(1.6)$ & $0.6(0.5)$ \\
VCAM-1 & $2.2(0.9)$ & $1.4(1.2)$ \\
Inflammation score & $9.3(1.0)$ & $5.2(2.6)$ \\
\hline
\end{tabular}

Sections of synovial tissue taken before treatment (before infliximab) and four weeks after the first day of treatment (after infliximab) were stained with the appropriate antibody and scored from 0 (minimum) to 4 (maximum) for CD $3+$ cells, $\mathrm{E}$ selectin, ICAM-1 and VCAM-1. The inflammation score was determined by the sum of scores of hyperplasia of the synovial lining, and infiltration with lymphocytes, plasma cells or polymorphonuclear cells. Data shown as mean (SD). Table derived partly from data published in Arthritis Rheum 1996; 39:1077-81 and reproduced by kind permission of the editor. 

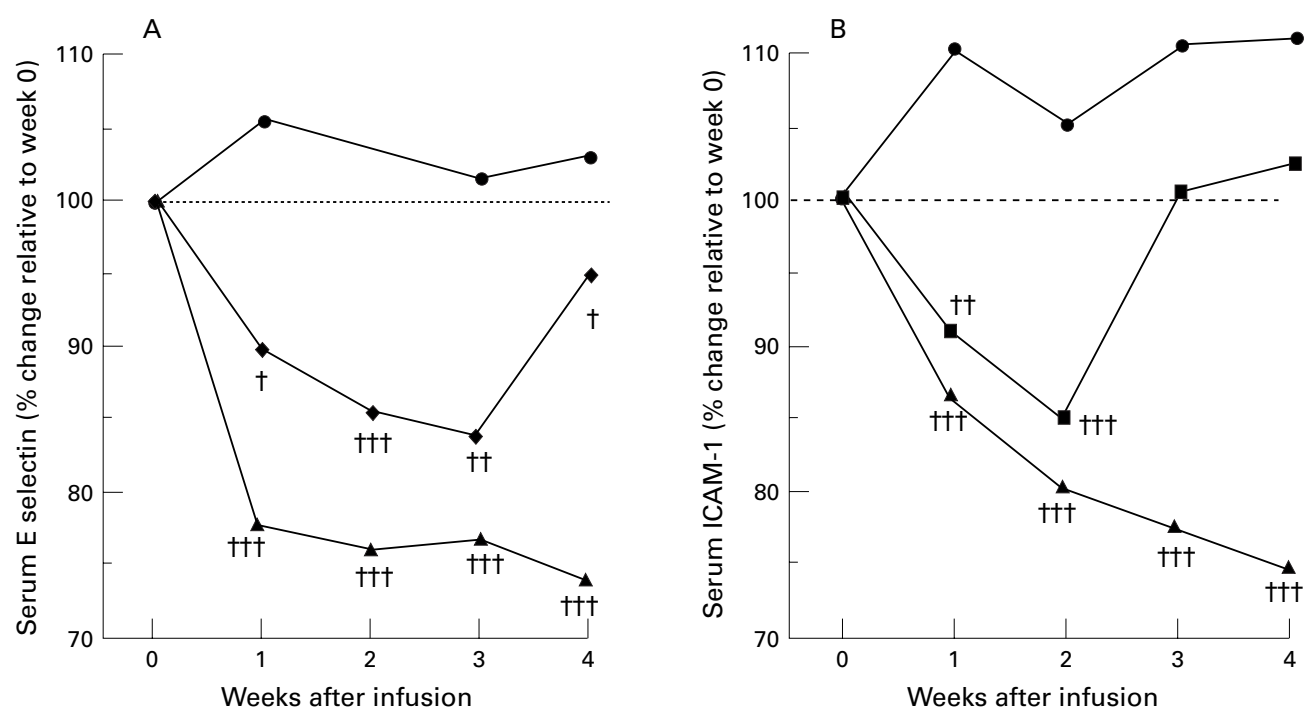

Figure 3 Decreases in (A) serum E selectin concentration and (B) serum ICAM-1 concentration after infliximab infusion in a randomised placebo controlled trial of infliximab in $R A$. Patients were treated on day 0 with a single, two hour infusion of either placebo (circle), $1 \mathrm{mg} / \mathrm{kg}$ infliximab (diamond) or $10 \mathrm{mg} / \mathrm{kg}$ infliximab (triangle). Values are expressed relative to median pre-infusion values (100\%) in placebo treated $(n=24), 1 \mathrm{mg} / \mathrm{kg}(n=23)$ and $10 \mathrm{mg} / \mathrm{kg}(n=21)$ of infliximab treated patients. Significance was determined versus change in placebo treated group at different time points by Mann-Whitney U test: $t=p<0.05 ;+t=p<0.01$; $t+t=p<0.001$. Reproduced with modification from Arthritis Rheum 1996;39:1082-91 by kind permission of the editor

been further substantiated and a reduction in chemokines has been observed, for example, in the serum concentrations and synovial tissue expression of IL8 and MCP1 in patients whose pre-treatment blood and synovial tissue levels were significantly increased. ${ }^{6}$

\section{Deactivation of endothelium and alteration in trafficking patterns}

Reduction in the swelling and tenderness of RA joints after infliximab treatment prompted the investigation of joint pathology by serial synovial biopsies performed before and after the administration of infliximab. These studies have show clear evidence of the following:

- Reduction in the cellularity and inflammatory score of synovial tissues. ${ }^{7}$ This change

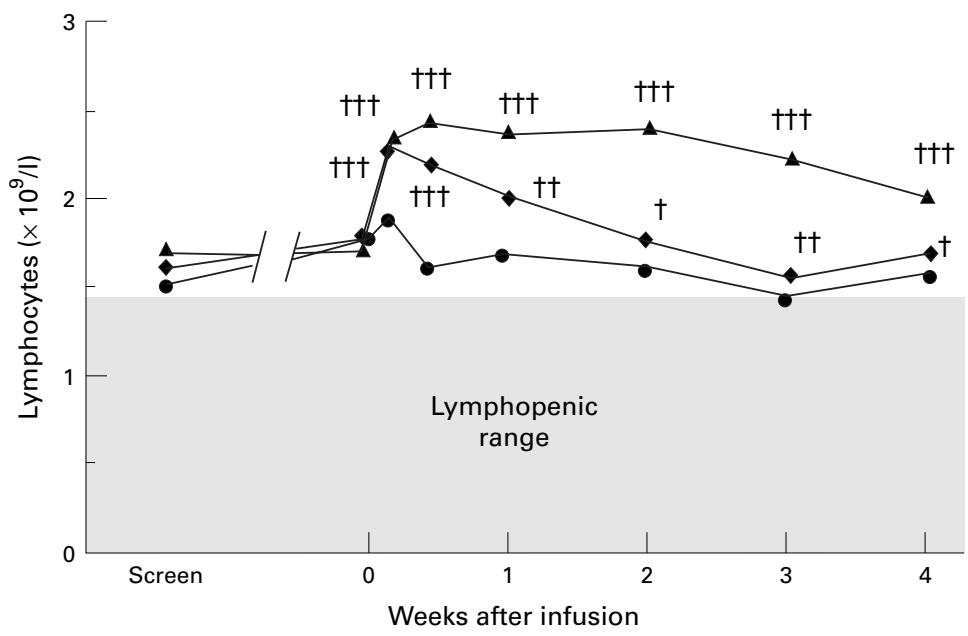

Figure 4 Changes in circulating lymphocyte counts after infliximab infusion in a randomised placebo controlled trial of infliximab in $R A$. Values are the median counts in placebo treated (circle) $(n=24), 1 \mathrm{mg} / \mathrm{kg}$ (diamond) $(n=23)$ and $10 \mathrm{mg} / \mathrm{kg}$ (triangle) $(n=21)$ of infliximab treated patients. Circulating numbers of lymphocytes in patients with active $R A$ were measured before and after treatment. Significance was versus change in placebo treated group at different time points by Mann-Whitney $U$ test: $\dagger=p<0.05 ; \dagger$ $=p<0.01 ;+t \dagger=p<0.001$. Reproduced with modification from Arthritis Rheum 1996;39:1082-91 by kind permission of the editor. can be largely attributed to a reduction in the number of $\mathrm{CD}^{+}$lymphocytes and $\mathrm{CD} 68^{+}$ cells derived from the monocyte lineage. ${ }^{7}$ (table 1).

- It has been found that the expression of cytokine induced vascular adhesion molecules (E selectin, ICAM-1 and VCAM-1) are all significantly reduced after infliximab. This conclusion is supported by the observed fall in serum concentrations of the soluble forms of E selectin and ICAM-1 (fig 3). ${ }^{8}$ Direct evidence of deactivation of endothelium is provided by immunohistological examination of biopsy specimens before and after treatment with infliximab that shows reduction in the expression of ICAM-1, VCAM-1 and E selectin (table 1).

- That the effect of these changes leads to reduction in the trafficking of lymphocytes is supported by the observed increase in the circulating numbers of lymphocytes in the blood of patients immediately after infliximab treatment (fig 4 ). ${ }^{8}$

We have concluded that neutralisation of $\mathrm{TNF} \alpha$ by infliximab in RA appears to down regulate the cytokine cascade and thus deactivates endothelium. We believe that the deactivation of endothelium reduces interactions between endothelial adhesion molecules and the counter ligands expressed on leucocytes, and consequently reduces the retention of circulating leucocytes in inflamed joints. In a microenvironment in which chemokines have also been down regulated, we postulate that the net influx into cells of the joints is consequently reduced. This ultimately leads to debulking of the inflammatory cells at the site of disease.

To test the hypothesis that circulating leucocytes show altered trafficking patterns, polymorphonuclear cells have been isolated from RA patients with active disease, who have knee and wrist synovitis, labelled with indium ${ }^{111}$, and 
reinjected into blood. Gammacamera scanning shows retention of labelled cells at these sites before infliximab treatment. However, when the experiment is repeated two weeks after a single intravenous injection of infliximab at 10 $\mathrm{mg} / \mathrm{kg}$ a clear cut reduction in radioisotope uptake over the knee joints and wrist joints is observed, thus directly confirming the reduction of retention of leucocytes in inflamed joints. ${ }^{9}$

\section{TNF $\alpha$ regulates angiogenesis}

More recently we have begun to examine the possibility that reduction in the inflammatory mass of tissue in rheumatoid joints after anti-TNF treatment may result from additional effects on the formation of new blood vessels. Angiogenesis is a feature of rheumatoid

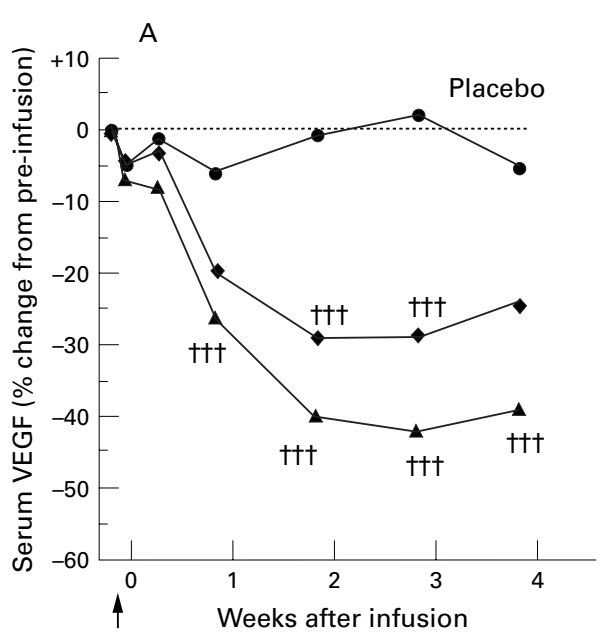

inflammation and is a process that is not only implicated in the enhanced delivery of inflammatory cells and mediators to the joint, but also in the invasive property of pannus at the cartilage and bone junction. In an unpublished study on 10 RA patients, we have been measuring angiogenesis by enumerating the number of blood vessels per unit area of microscopic sections of synovial membrane biopsy specimens, before and after anti-TNF treatment, for the expression of $\alpha \mathrm{V} \beta 3$ and CD31 on vascular endothelium. Preliminary analysis suggests that newly formed blood vessels are reduced after infliximab treatment. ${ }^{10}$ We can conclude that an additional factor under the control of $\mathrm{TNF} \alpha$, and revealed by $\mathrm{TNF} \alpha$ blockade, is a reduction of angiogenesis. That this reduction in angiogenesis is likely to be a

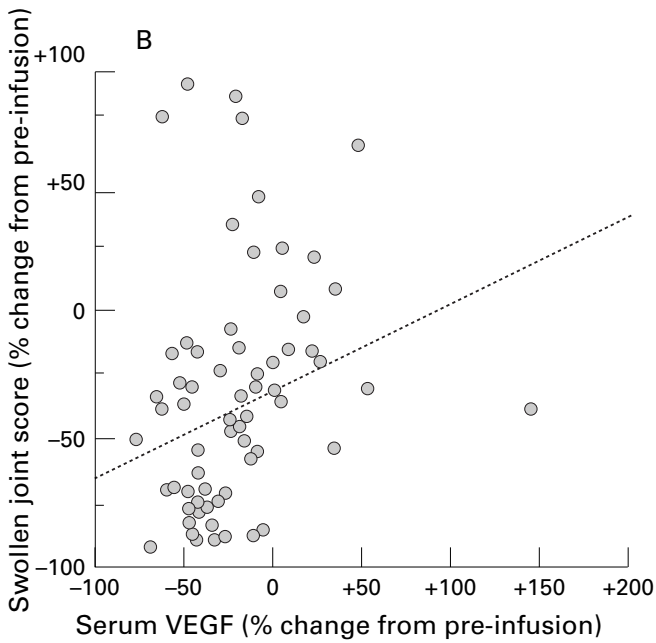

Figure 5 (A) Decrease in serum VEGF concentration after a single infusion of infliximab in a randomised placebo controlled trial of infliximab in RA. Serum VEGF levels were measured by enzyme linked immunosorbent assay in 69 patients with active $R A$ who received a single infusion of either placebo or infliximab (indicated by arrow). Values are the median counts in placebo treated (circle), $1 \mathrm{mg} / \mathrm{kg}$ (diamond) and $10 \mathrm{mg} / \mathrm{kg}$ (triangle) of infliximab treated patients. Values were expressed as the \% change from preinfusion for each patient, before calculation of median changes for each treatment group. Data were analysed by Mann-Whitney $U$ test for comparison between treatment groups $(\dagger=p<0.05 ;+\dagger$ $=p<0.01 ; \mathrm{H}+\mathrm{t}=p<0.001)$. $p$ Values for comparisons between multiple groups were adjusted using the Bonferroni correction. (B) Correlation between changes in serum VEGF concentrations and changes in swollen joint scores. Kendall's coefficient of rank correlation, calculated using \% change in serum VEGF and swollen joint scores three weeks after infusion of infliximab is $0.245(p<0.01)$. Reproduced with modification from Arthritis Rheum 1998;41:1258-65 by kind permission of the editor.
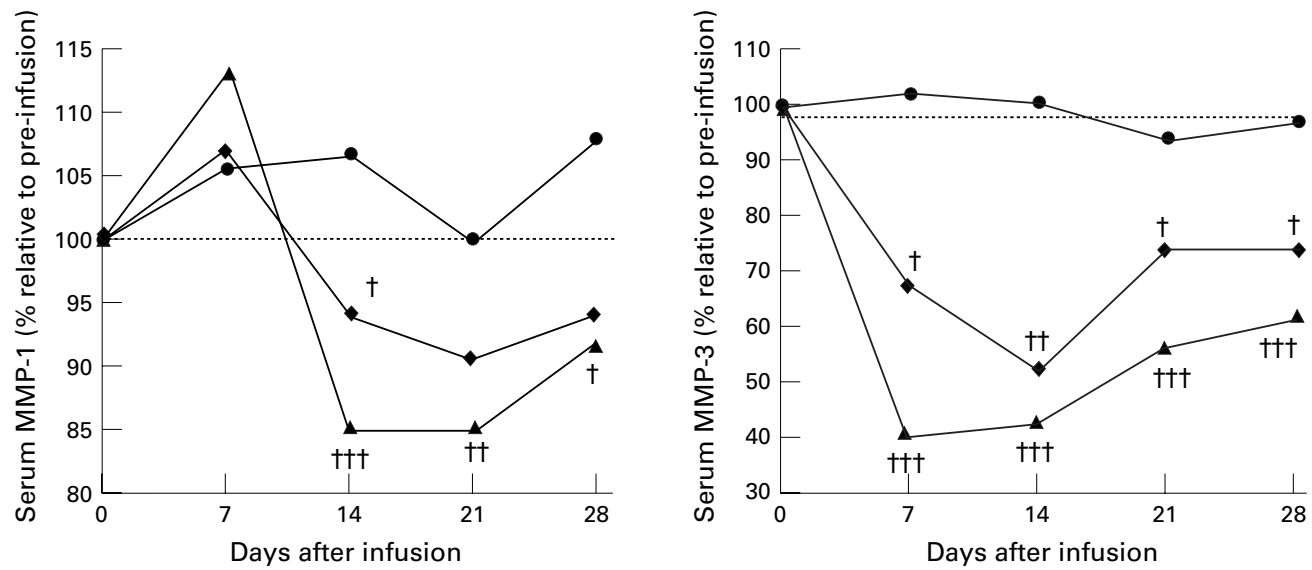

Figure $6 \quad M M P-1$ and $M M P-3$ levels are decreased after infliximab treatment in a randomised placebo controlled trial of infliximab in RA. MMP-1 and MMP-3 levels were determined in serum by a double antibody sandwich ELISA in placebo (circle) $(n=21), 1 \mathrm{mg} / \mathrm{kg}$ (diamond) $(n=19), 10 \mathrm{mg} / \mathrm{kg}$ (triangle) $(n=17)$ infliximab treated RA patients before infusion (day 0) and 7, 14,21 and 28 days after infusion. Values are expressed relative to pre-infusion values (100\%). Statistical analysis between the infliximab treated groups and placebo at day 7, 14, 21 and 28 was performed using the Mann-Whitney $U$ test $(t=p<0.05 ;+t=p<0.01 ;+t+=p<0.001)$. Reproduced with modification from Br $\mathcal{F}$ Rheumatol 1997;36:643-50 by kind permission of the editor. 
result of the regulation of endothelial growth factors by $\mathrm{TNF} \alpha$ is suggested by the reduction in raised concentrations of serum vascular endothelial growth factor (VEGF) after infliximab (fig 5). ${ }^{11}$

\section{TNF $\alpha$ and tissue destruction}

While anti-TNF treatment in the murine collagen induced arthritis model was found to protect joints from damage, ${ }^{12}$ it is not yet established that anti-TNF treatment protects rheumatoid joints from structural damage. Indirect measurements of surrogate markers of joint destruction have been performed by us in short-term trials of infliximab. Measurement of circulating matrix metalloproteinases (MMP1 and MMP3) in their inactive form has been noted to be reduced after infliximab in a dose related fashion (fig 6). ${ }^{13}$ Taken in conjunction with a reduction in inflammation, angiogenesis and other signs of disease activity, which are usually linked to tissue damage, these data predict that anti-TNF treatment might slow down the destructive process in RA joints. In the current ongoing trial of infliximab treatment, the 54 week end point will examine the progression of erosions and joint space narrowing on serial radiographs before and after anti-TNF treatment to establish whether infliximab indeed has any disease modifying capacity.

\section{Conclusion}

In this brief communication we have summarised the experimental data of effects of infliximab treatment on the rheumatoid disease process. Our observations indicate that TNF $\alpha$ plays a key part in regulating the cytokine network, and its biological effects. An important aspect is the induction of cell adhesion and cell migration into rheumatoid joints. Furthermore, angiogenesis is induced by TNF. These observations explain the anti-inflammatory effects of TNF blockade and predict that the destructive effects of the disease should be ameliorated by such treatments.

Clinical trials have established that long term continuing treatment with anti-TNF agents is required to control rheumatoid disease. At this point it is unclear whether this treatment strategy will be associated with any undesirable consequences, for example, by host immunity being compromised. Experimental evidence suggests that TNF does play an important part in host defence mechanisms against certain infections with organisms such as listeria, mycobacteria and pathogenic protozoa. Long term observations in the clinic will be required to establish whether other molecular and cellular processes can compensate for loss of this defence mechanism. An interesting, and as yet unanswered, twist to this discussion is the possibility that the immunoregulatory consequences of neutralising $\mathrm{TNF} \alpha$ with specific monoclonal antibodies, such as infliximab, may not be identical to treating patients with soluble TNF receptor immunoglobulin fusion proteins such as etanercept, which neutralise not only $\mathrm{TNF} \alpha$, but also lymphotoxin. In RA it is believed that $\mathrm{TNF} \alpha$ is produced in excess while little or no lymphotoxin is simultaneously produced. ${ }^{14}$ Thus we have assumed that blockade of TNF by infliximab and etanercept will have an identical efficacy and safety profile. Application in the clinic will ultimately reveal whether this assumption is justified.

The Kennedy Institute of Rheumatology is supported by the Arthritis Research Campaign, UK. We thank Ms Lindsay Roffe and Ms Annette Winter for assistance with preparation of the manuscript.

1 Feldmann M, Bondeson J, Brennan FM, Foxwell BMJ, Maini RN. The rationale for the current boom in anti-TNF $\alpha$ treatment. Is there an effective means to define therapeutic targets for drugs that provide all the benefits of anti-TNF $\alpha$ and minimise hazards? Ann Rheum Dis 1999;58:(Suppl I)I27-I31

2 Elliott MJ, Maini RN, Feldmann M, et al. Treatment of rheumatoid arthritis with chimeric monoclonal antibodies to tumor necrosis factor $\alpha$. Arthritis Rheum 1993; 36:1681-90.

3 Elliott MJ, Maini RN, Feldmann M, et al. Randomised double-blind comparison of chimeric monoclonal antibody to tumour necrosis factor $\alpha$ (cA2) versus placebo in to tumour necrosis factor $\alpha$ (cA2) versus place
rheumatoid arthritis. Lancet 1994; 344:1105-10.

4 Charles P, Elliott MJ, Davis D, et al. Regulation of cytokines, cytokine inhibitors and acute phase proteins following anti$\mathrm{TNF} \alpha$ therapy in rheumatoid arthritis. J Immunol TNFa therapy in

5 Lorenz HM, Antoni C, Valerius T, et al. In vivo blockade of TNF- $\alpha$ by intravenous infusion of a chimeric monoclonal TNF- $\alpha$ antibody in patients with rheumatoid arthritis. Short term cellular and molecular effects. J Immunol 1996; 156:1646-53.

6 Taylor PC, Chapman P, Elliott MJ, et al. TNF $\alpha$ blockade in patients with rheumatoid reduces granulocyte margination and traffic to joints with concomitant reduction in synovial chemotactic gradients. [Abstract]. Clin Exp Rheumatol 1998;16:204.

7 Tak PP, Taylor PC, Breedveld FC, et al. Decrease in cellularity and expression of adhesion molecules in rheumatoid synovial tissue after anti-TNF $\alpha$ monoclonal antibody treatment. Arthritis Rheum 1996;39:1077-81.

8 Paleolog EM, Hunt M, Elliott MJ, Woody JN, Feldmann M, Paleolog EM, Hunt M, Elliott MJ, Woody JN, Feldmann M,
Maini RN. Deactivation of vascular endothelium by monoMaini RN. Deactivation of vascular endothelium by mono-
clonal anti-tumor necrosis factor $\alpha$ antibody in rheumatoid clonal anti-tumor necrosis factor $\alpha$ antibody in
arthritis. Arthritis Rheum 1996;39:1082-91.

9 Taylor PC, Chapman P, Elliott MJ, et al. Reduced granulocyte traffic and chemotactic gradients in rheumatoid joints following anti-TNF- $\alpha$ antibody therapy. Arthritis Rheum (in press).

10 Ballara S, Taylor PC, Paleolog E, Feldmann M, Maini RN. $\mathrm{TNF} \alpha$ blockade in rheumatoid arthritis reduces synovial vascularity. [Abstract]. Scand J Immunol 1999;49:333.

11 Paleolog EM, Young S, Stark AC, et al. Modulation of angiogenic vascular endothelial growth factor by tumor necrosis factor $\alpha$ and interleukin-1 in rheumatoid arthritis. Arthritis Rheum 1998;41:1258-65.

12 Williams RO, Feldmann M, Maini RN. Anti-Tumor necrosis factor ameliorates joint disease in murine collageninduced arthritis. Proc Nat Acad Sci USA 1992;89: 9784-8.

13 Brennan FM, Browne KA, Green PA, Jaspar J-M, Maini Brennan FM, Browne KA, Green PA, Jaspar J-M, Maini
RN, Feldmann M. Reduction of serum matrix metalloproteinase 1 and matrix metalloproteinase 3 in rheumatoid teinase 1 and matrix metalloproteinase 3 in rheumatoid
arthritis patients following anti-tumour necrosis factor- $\alpha$ (cA2) therapy. Br J Rheumatol 1997;36:643-50.

14 Brennan FM, Chantry D, Jackson A, Maini RN, Feldmann M. Inhibitory effect of TNF alpha antibodies on synovial cell interleukin-1 production in rheumatoid arthritis. Lancet $1989 ; 2: 244-7$ 